\title{
(2) OPEN ACCESS \\ Association of education levels with the risk of hypertension and hypertension control: a nationwide cohort study in Chinese adults
}

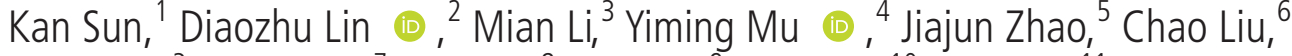 \\ Yufang Bi, ${ }^{3}$ Lulu Chen, ${ }^{7}$ Lixin Shi, ${ }^{8}$ Qiang Li, ${ }_{1}^{9}$ Tao Yang, ${ }^{10}$ Qin Wan, ${ }^{11}$ Shengli Wu, ${ }^{12}$ \\ Guixia Wang, ${ }_{13}$ Zuojie Luo, ${ }^{14}$ Yingfen Qin, ${ }^{14}$ Xulei Tang, ${ }^{15}$ Gang Chen (ㄷ), ${ }^{16}$ \\ Yanan Huo, ${ }^{17}$ Zhengnan Gao, ${ }^{18}$ Qing Su, ${ }_{19}^{19}$ zhen $\mathrm{Ye}_{1}{ }^{20}$ Ruying $\mathrm{Hu}_{1}{ }^{21}$ Youmin Wang, ${ }^{22}$ \\ Guijun Qin, ${ }^{23}$ Huacong Deng, ${ }^{24}$ Xuefeng Yu, ${ }^{25}$ Feixia Shen, ${ }^{26}$ Li Chen, ${ }^{27}$ \\ Weiqing Wang, ${ }^{28}$ Guang Ning, ${ }^{28}$ Li Yan ${ }^{29}$
}

\begin{abstract}
- Additional supplemental material is published online only. To view, please visit the journal online (http://dx doi.org/10.1136/jech-2021217006).
\end{abstract}

For numbered affiliations see end of article.

Correspondence to Professor Li Yan, Department of Endocrinology, Sun Yat-Sen Memorial Hospital, Guangzhou, Guangdong, China; hfxyl@163.net

Received 13 April 2021 Accepted 8 November 2021
D) Check for updates

(C) Author(s) (or their employer(s)) 2022. Re-use permitted under CC BY-NC. No commercial re-use. See rights and permissions. Published by BMJ.

To cite: Sun K, Lin D, Li M et al. J Epidemiol Community Health Epub ahead of print: [please include Day Month Year]. doi:10.1136/jech2021-217006

\begin{abstract}
Background Education attainment can improve life expectancy and guide healthy behaviours throughout an entire lifetime. A nationwide longitudinal study of the association of education status with the risk of hypertension and its control in China is lacking.

Methods The China Cardiometabolic Disease and Cancer Cohort Study is a multicentre, population-based, prospective cohort study. We performed the baseline survey from 2011 to 2012. A follow-up visit was conducted during 2014-2016. 101959 subjects were included in the final data analyses. Cox proportional hazards regression was used to examine the associations of education levels with the risk of hypertension and uncontrolled hypertension.
\end{abstract}

Results During follow-up, 11189 (19.9\%) participants had developed hypertension among subjects without hypertension at baseline. Among the participants with hypertension at baseline, only $40.6 \%$ had controlled hypertension. Compared with the participants' education level at elementary school and below, the multivariableadjusted $\mathrm{HR}$ for incident hypertension was $0.76(95 \% \mathrm{Cl}$, 0.72 to 0.80 ) in those with a middle school education level and $0.67(95 \% \mathrm{Cl}, 0.63$ to 0.70$)$ in those with a high school degree or above. Correspondingly, multivariable-adjusted HRs associated with uncontrolled hypertension were $0.90(95 \% \mathrm{Cl}, 0.87$ to 0.92$)$ in participants with a middle school education level and $0.85(95 \% \mathrm{Cl}, 0.82$ to 0.88$)$ in participants with a high school degree or above level.

Conclusion Participants with education attainment at elementary school and below exhibited excess risks of newly diagnosed hypertension and worse blood pressure control compared with individuals with education attainment at middle school or above.

\section{INTRODUCTION}

As the leading cause of adverse cardiovascular outcomes, hypertension (HTN) remains a worldwide burden in most economically developing and developed countries. ${ }^{1}$ The social determinants of health $(\mathrm{SDOH})$ partly explain the high burden of HTN and related diseases in developing countries. $^{2-4}$ Compared with other determinants, such as income level or social status, education has taken centre stage among $\mathrm{SDOH}$, affecting people throughout their entire lifetimes by improving life expectancy and guiding healthy behaviours. ${ }^{5}$

As recently reported in the Prospective Urban Rural Epidemiologic study, low-income individuals with low educational levels have a lower prevalence of HTN than those with higher education attainment. ${ }^{6}$ Inconsistent with this finding, in the same study, high-income individuals with high levels of education are more likely to be health-conscious and therefore have a markedly reduced prevalence of HTN. Differences in the relationship between educational groups and HTN prevalence can be explained by markedly poorer healthcare according to the level of education and income. ${ }^{78}$ Additionally, individuals in low-income countries and with lower levels of education have received poorer secondary prevention and poorer therapy for HTN than other groups. ${ }^{9}$

The effect of fast-paced economic growth on $\mathrm{SDOH}$ changes has resulted in a rapid increase in the incidence of HTN in China. ${ }^{10}$ With the resumption of college entrance examination in 1977 and the reform and opening-up policy in 1978, the education level and lifestyle of Chinese individuals aged 40 years and older have been significantly different. The prevalence of HTN in individuals aged 40 years and older has increased significantly in China. The link between education and HTN has been examined in previous studies. ${ }^{11-13}$ The influence of education is wide, not only in the lifestyle of the subjects but also in their medication compliance and frequency of blood pressure selfmonitoring after a diagnosis of HTN. According to previous studies, a low education level may directly lead to the increased incidence of HTN and may also directly affect the blood pressure control of patients with previous HTN. However, although a proportion of the previous studies comprised longitudinal studies, they only included some specific ages and some regions and the sample sizes were not sufficiently large. According to previous studies, we considered that the education level is related to the incidence and control of HTN, but this finding must be confirmed in larger samples of the Chinese 


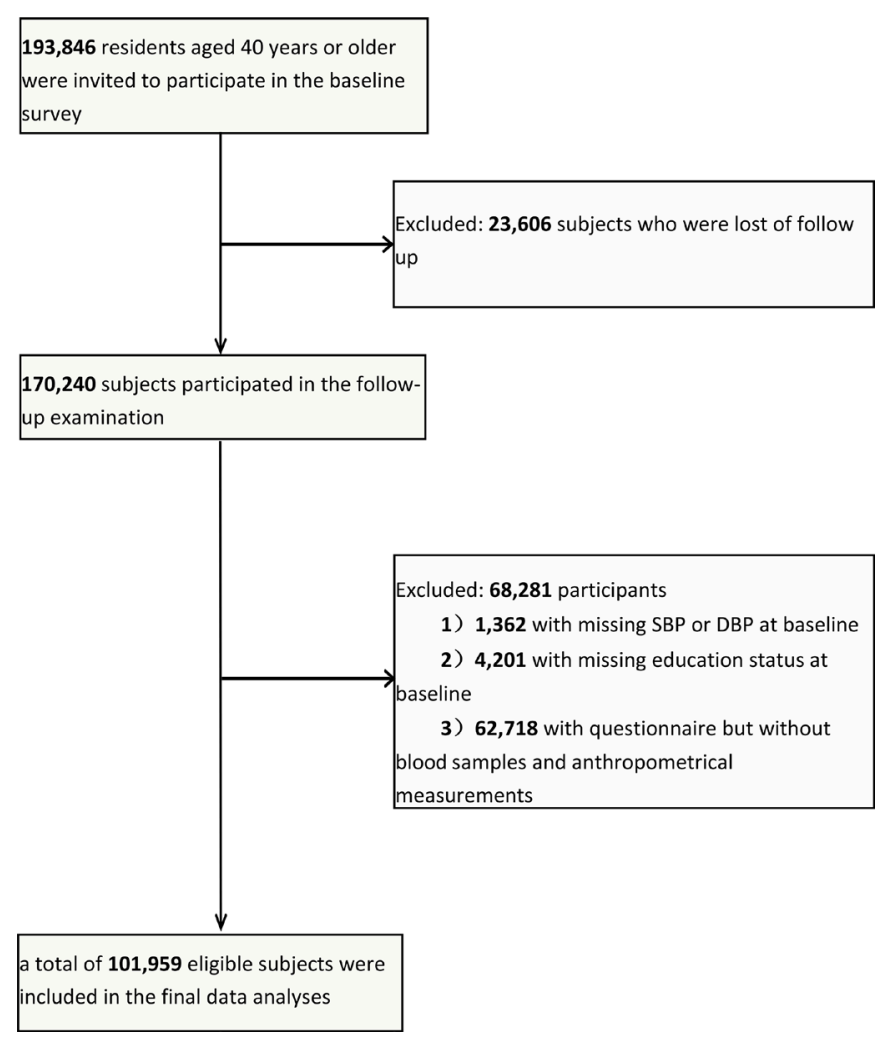

Figure 1 Flowchart of the population selection of the study. DBP, diastolic blood pressure; SBP, systolic blood pressure.

population, particularly in those aged 40 years and older and including both high-income and low-income or middle-income communities. Therefore, we performed the present cohort study in a large nationwide Chinese population to evaluate the effects of education levels on the incidence of HTN and blood pressure control in subjects with previously diagnosed HTN.

\section{METHODS}

\section{Study population and study design}

The study participants were obtained from the China Cardiometabolic Disease and Cancer Cohort (4C) Study, and details of this cohort have been published previously. ${ }^{14}{ }^{15}$ From 2011 to 2012 , 259657 individuals aged $\geq 40$ years were recruited from 25 communities throughout various regions of China. However, in 2014, because of limited funds, only 193846 participants from 20 communities from various geographical regions in China, selected to represent the general population, were invited to participate. From 2014 to 2016, 170240 participants completed the follow-up examination visit, and the follow-up rate of the study was $87.8 \%$. Participants who failed to provide information on education and blood pressure were excluded from the analyses. To evaluate the effects of education levels on the incidence of HTN and blood pressure control in subjects with previously diagnosed HTN, subjects with previously diagnosed HTN at baseline were also enrolled. Finally, 101959 subjects were included in the final data analyses. The detailed processes of the study population are presented in a flow diagram figure 1. Before data collection, each participant had provided written informed consent.

\section{Questionnaire and biochemical investigation}

Information on sociodemographic characteristics, lifestyle factors and family history were collected using a standardised questionnaire at baseline and the follow-up visit. Data collection and anthropometric measurements were conducted by clinical staff members in local community clinics who were trained and certified. Education attainment was self-reported and categorised as follows: (1) elementary school and those below elementary school, (2) middle school and (3) high school degree or above. Smoking regularly in the past 6 months was classified as current smokers, while other participants were classified as non-current smokers. Drinking regularly in the past 6 months was classified as current drinkers, while other participants were classified as noncurrent drinkers. Physical activity was estimated by the International Physical Activity Questionnaire, according to questions on the frequency and duration of moderate or vigorous activities and walking. ${ }^{16}$ Ideal physical activity was defined as $\geq 75 \mathrm{~min} /$ week of vigorous intensity or $\geq 150 \mathrm{~min} /$ week of moderate and vigorous intensity or $\geq 150 \mathrm{~min} /$ week of moderate intensity.

Body weight and height were, respectively, measured to the nearest $0.1 \mathrm{~kg}$ and $0.1 \mathrm{~cm}$. Body mass index (BMI) was calculated as follows: weight in kilograms/(height in metres) ${ }^{2}$. Obesity was defined as a BMI $\geq 30 \mathrm{~kg} / \mathrm{m}^{2}$, and overweight was defined as a BMI $\geq 25 \mathrm{~kg} / \mathrm{m}^{2}$ and $<30 \mathrm{~kg} / \mathrm{m}^{2}$. Venous blood samples were collected after fasting for at least 10 hours. Fasting plasma glucose (FPG), fasting insulin, creatinine, $\gamma$-glutamyltransferase $(\gamma$-GGT), total cholesterol (TC), triglycerides (TG), high-density lipoprotein cholesterol (HDL-C) and low-density lipoprotein cholesterol (LDL-C) were evaluated using the autoanalyser (Beckman CX-7 Biochemical Autoanalyser; Brea, California, USA). Homeostasis model assessment of insulin resistance (HOMA-IR), which measures the Insulin Resistance Index, was calculated as follows: fasting insulin $(\mathrm{mIU} / \mathrm{mL}) \times$ fasting glucose $(\mathrm{mmol} / \mathrm{L}) / 22.5{ }^{17}$ To calculate the estimated glomerular filtration rate (eGFR), we used the following abbreviated Modification of Diet in Renal Disease formula recalibrated for the Chinese population: eGFR $\left(\mathrm{mL} / \mathrm{min}\right.$ per $\left.1.73 \mathrm{~m}^{2}\right)=175 \times$ (serum creatinine $(\mu \mathrm{mol} / \mathrm{L}) \times 0.011)^{-1.234} \times(\text { age })^{-0.179} \times(0.79$ if woman $){ }^{18}$

\section{Definition of newly diagnosed HTN and uncontrolled HTN}

Three consecutive blood pressure measurements within a 5 -min interval were obtained from each participant using an automated electronic device (OMRON; Omron Company, China). The average of three measurements of systolic blood pressure (SBP) and diastolic blood pressure (DBP) were used for analysis. Newly diagnosed HTN was defined as SBP $\geq 140 \mathrm{mmHg}$ and/or $\mathrm{DBP} \geq 90 \mathrm{mmHg}$ and/or currently taking antihypertension medication during the follow-up visit in subjects without HTN at baseline. ${ }^{19}$ Uncontrolled HTN was defined as SBP $\geq 140 \mathrm{mmHg}$ and/or DBP $\geq 90 \mathrm{mmHg}$ during the follow-up visit in subjects with HTN at baseline. ${ }^{20}$

\section{Statistical analysis}

Continuous variables were presented as means $\pm S D$, while skewed variables were presented as medians (IQRs). Categorical variables are expressed as numbers (proportions). Differences in clinical characteristics and laboratory variables among groups were tested by one-way analysis of variance. $\mathrm{X}^{2}$ test was used to compare categorical variables. Trends across different education group levels were evaluated using linear regression analyses. We used Cox proportional hazards regression models to assess the risk of newly diagnosed HTN events and uncontrolled HTN events according to individual education levels. HRs and the corresponding 95\% CIs were calculated. The covariates in fully adjusted Cox proportional hazards regression models were generated according to previous publications and potential 
intermediate factors related to the development and progression of HTN. Model 1 was unadjusted. Model 2 was adjusted for age. Model 3 was further adjusted for BMI and sex. Model 4 was further adjusted for current drinking, current smoking, a family history of diabetes, creatinine, GGT, FPG, insulin, LDL-C and TG. Model 5 was further adjusted for baseline SBP and DBP. In subgroup analyses, the associations of education levels with newly diagnosed HTN and uncontrolled HTN were stratified by median age $(\geq 56.5 /<56.5$ years), sex (men/women), BMI (normal/overweight/obesity), diabetes status (yes/no), current drinking status (yes/no) and current smoking status (yes/no). The interaction tests were performed by simultaneously including the interaction terms (strata variable multiplied by HTN status), each strata factor and HTN status in the Cox regression analyses.

SAS V.9.3 (SAS Institute) was used to perform statistical analysis. All statistical tests were two-sided, and a $p$ value $<0.05$ was indicated statistical significance.

\section{RESULTS}

\section{Clinical characteristics of the cohort}

During a 3.8-year follow-up period, 11189 (19.9\%) subjects developed HTN among the 56151 subjects without HTN at baseline. Among participants with HTN at baseline, 27221 (59.4\%) participants had uncontrolled HTN during the follow-up visit. The participants' characteristics according to the HTN status are reported in table 1. Compared with participants without HTN at baseline and follow-up stage (non-HTN group), those with HTN at baseline or follow-up were older, had a higher BMI, waist circumference, FPG, fasting insulin, HOMA-IR, TC, TG, LDL-C, alanine aminotransferase, aspartate aminotransferase and $\gamma$-GGT, and were more likely to be a current drinker (all $p<0.0001)$. In addition to the difference in investigation and biochemical measurements among groups, a larger proportion of subjects in the non-HTN group had attained higher education levels than in the other two groups $(p<0.0001)$. Compared with participants with an elementary school education level and below, those with higher education levels exhibited a significantly lower HTN prevalence and decreased SBP and DBP at baseline (table 2; all $p$ for trend $<0.0001$ ).

\section{Associations of the education level with newly diagnosed HTN and uncontrolled HTN}

The incidences of newly diagnosed HTN in the elementary school and below group, middle school group and high school

Table 1 Baseline characteristics of the study population at follow-up according to hypertension (HTN) status

\begin{tabular}{|c|c|c|c|c|}
\hline & Non-HTN & HTN at follow-up & HTN at baseline & $P$ value \\
\hline $\mathrm{n}(\%)$ & $44962(44.10)$ & $11189(10.97)$ & 45808 (44.93) & -- \\
\hline Age (years) & $53.87 \pm 8.06$ & $56.83 \pm 8.57^{*} \dagger$ & $59.45 \pm 8.46^{*}$ & $<0.0001$ \\
\hline Male (n (\%)) & $13223(29.41)$ & $4082(36.48) * \dagger$ & $17504(38.21)^{*}$ & $<0.0001$ \\
\hline BMI $\left(\mathrm{kg} / \mathrm{m}^{2}\right)$ & $23.71 \pm 3.35$ & $24.74 \pm 3.54^{*} \dagger$ & $25.50 \pm 3.55^{*}$ & $<0.0001$ \\
\hline WC (cm) & $81.25 \pm 9.19$ & $84.52 \pm 9.59 * t$ & $86.31 \pm 9.73^{*}$ & $<0.0001$ \\
\hline $\mathrm{SBP}(\mathrm{mmHg})$ & $118.24 \pm 11.25$ & $127.18 \pm 8.95^{*} \dagger$ & $149.67 \pm 17.67^{*}$ & $<0.0001$ \\
\hline $\mathrm{DBP}(\mathrm{mmHg})$ & $72.40 \pm 7.75$ & $76.73 \pm 7.56^{*} \dagger$ & $85.13 \pm 10.95^{*}$ & $<0.0001$ \\
\hline Current smoking (n (\%)) & $6434(14.31)$ & $1849(16.53)^{*} \dagger$ & $6460(14.10)^{*}$ & $<0.0001$ \\
\hline Current drinking (n (\%)) & $3863(8.59)$ & $1283(11.47)^{*} \dagger$ & $5550(12.12)^{*}$ & $<0.0001$ \\
\hline TG (mmol/L) & $1.19(0.86-1.71)$ & $1.35(0.96-1.95)^{*} \dagger$ & $1.47(1.04-2.13)^{*}$ & $<0.0001$ \\
\hline $\mathrm{TC}(\mathrm{mmol} / \mathrm{L})$ & $4.85 \pm 1.13$ & $4.95 \pm 1.13^{*} \dagger$ & $5.10 \pm 1.12^{*}$ & $<0.0001$ \\
\hline $\mathrm{HDL}-\mathrm{C}(\mathrm{mmol} / \mathrm{L})$ & $1.35 \pm 0.37$ & $1.33 \pm 0.36^{*}$ & $1.33 \pm 0.36^{*}$ & $<0.0001$ \\
\hline LDL-C (mmol/L) & $2.81 \pm 0.86$ & $2.86 \pm 0.86^{*} \dagger$ & $2.96 \pm 0.88^{*}$ & $<0.0001$ \\
\hline FPG (mmol/L) & $5.71 \pm 1.38$ & $6.02 \pm 1.71^{*} \dagger$ & $6.20 \pm 1.74^{*}$ & $<0.0001$ \\
\hline Fasting insulin ( $\mu \mathrm{lU} / \mathrm{ml})$ & $6.20(4.40-8.50)$ & $6.80(4.80-9.60)^{*} \dagger$ & $7.50(5.20-10.60)^{*}$ & $<0.0001$ \\
\hline $\operatorname{ALT}(\mathrm{U} / \mathrm{L})$ & $14.00(11.00-20.00)$ & $15.40(11.00-22.00)^{*} \dagger$ & $16.00(12.00-22.80)^{*}$ & $<0.0001$ \\
\hline AST (U/L) & $20.00(16.00-24.00)$ & $20.20(17.00-25.00)^{*} \dagger$ & $21.00(17.20-26.00)^{*}$ & $<0.0001$ \\
\hline$\gamma$-GGT (U/L) & $18.00(13.00-27.00)$ & $21.00(15.00-32.00)^{*} \dagger$ & $23.00(16.00-35.00)^{*}$ & $<0.0001$ \\
\hline eGFR $\left(\mathrm{mL} / \mathrm{min}\right.$ per $\left.1.73 \mathrm{~m}^{2}\right)$ & $98.13(89.97-104.54)$ & $95.85(87.19-102.49)^{*} \dagger$ & $92.93(83.72-99.67)^{*}$ & $<0.0001$ \\
\hline Ideal physical activity (n (\%)) & $6780(15.08)$ & $1397(12.49)^{*}$ & $5758(12.57)^{*}$ & $<0.0001$ \\
\hline HOMA-IR & $1.51(1.06-2.18)$ & $1.74(1.20-2.58)^{*} \dagger$ & $1.97(1.31-2.95)^{*}$ & $<0.0001$ \\
\hline Family history of diabetes ( $\mathrm{n}(\%)$ ) & $6318(14.05)$ & $1223(10.93)^{*} \dagger$ & $5469(11.94)^{*}$ & $<0.0001$ \\
\hline \multicolumn{5}{|l|}{ Education level (n (\%)) } \\
\hline Elementary school and below & $10005(22.25)$ & $3697(33.04)^{*} \dagger$ & $16322(35.63)^{*}$ & $<0.0001$ \\
\hline Middle school & $15964(35.51)$ & $3842(34.34)^{*} \dagger$ & $15491(33.82)^{*}$ & \\
\hline High school degree or above & $18993(42.24)$ & $3650(32.62)^{*} \dagger$ & $13995(30.55)^{*}$ & \\
\hline Use of antihypertensive medications ( $\mathrm{n}(\%)$ ) & - & - & $12913(28.19)$ & $<0.0001$ \\
\hline
\end{tabular}

Data were means \pm SD or medians (IQRs) for skewed variables or numbers (proportions) for categorical variables.

$P$ values were for analysis of variance or $\chi^{2}$ analyses across the groups.

${ }^{*} P<0.05$ compared with non-HTN group.

$+P<0.05$ compared with HTN at baseline group.

ALT, alanine aminotransferase ; AST, aspartate aminotransferase ; BMI, body mass index; DBP, diastolic blood pressure; eGFR, estimated glomerular filtration rate; FPG, fasting plasma glucose; HDL-C, high-density lipoprotein cholesterol ; HOMA-IR, homeostasis model assessment of insulin resistance ; LDL-C, low-density lipoprotein cholesterol ; SBP, systolic blood pressure; TC, total cholesterol; TG, triglycerides; WC, waist circumference; $\gamma$-GGT, $\gamma$-glutamyltransferase . 
Table 2 Baseline characteristics of study population at baseline by education level

\begin{tabular}{|c|c|c|c|c|}
\hline & Elementary school and below & Middle school & High school degree or above & $P$ for trend \\
\hline $\mathrm{n}(\%)$ & $30024(29.45)$ & $35297(34.62)$ & 36638 (35.93) & -- \\
\hline Prevalence of HTN (n (\%)) & $16322(54.36)$ & $15491(43.89)$ & $13995(38.20)$ & $<0.0001$ \\
\hline Age (years) & $60.09 \pm 8.78$ & $55.54 \pm 8.19$ & $55.05 \pm 5.36$ & $<0.0001$ \\
\hline Male $(\mathrm{n}(\%))$ & $8000(26.65)$ & $12873(36.47)$ & $13936(38.04)$ & $<0.0001$ \\
\hline BMI $\left(\mathrm{kg} / \mathrm{m}^{2}\right)$ & $24.69 \pm 3.68$ & $24.75 \pm 3.55$ & $24.46 \pm 3.48$ & $<0.0001$ \\
\hline WC (cm) & $83.78 \pm 10.06$ & $84.19 \pm 9.70$ & $83.66 \pm 9.61$ & 0.038 \\
\hline $\mathrm{SBP}(\mathrm{mmHg})$ & $138.99 \pm 21.91$ & $132.62 \pm 20.11$ & $129.10 \pm 19.23$ & $<0.0001$ \\
\hline $\mathrm{DBP}(\mathrm{mmHg})$ & $79.23 \pm 11.24$ & $79.10 \pm 11.04$ & $77.59 \pm 10.96$ & $<0.0001$ \\
\hline Current smoking ( $(\%))$ & $3783(12.60)$ & $6052(17.15)$ & $4908(13.40)$ & 0.13 \\
\hline Current drinking (n (\%)) & $3008(10.02)$ & $4074(11.54)$ & $3614(9.86)$ & 0.22 \\
\hline TG $(\mathrm{mmol} / \mathrm{L})$ & $1.31(0.93-1.90)$ & $1.34(0.95-1.95)$ & $1.32(0.94-1.93)$ & 0.51 \\
\hline $\mathrm{TC}(\mathrm{mmol} / \mathrm{L})$ & $5.02 \pm 1.13$ & $4.96 \pm 1.13$ & $4.96 \pm 1.14$ & $<0.0001$ \\
\hline $\mathrm{HDL}-\mathrm{C}(\mathrm{mmol} / \mathrm{L})$ & $1.38 \pm 0.37$ & $1.33 \pm 0.36$ & $1.31 \pm 0.35$ & $<0.0001$ \\
\hline $\mathrm{LDL}-\mathrm{C}(\mathrm{mmol} / \mathrm{L})$ & $2.88 \pm 0.87$ & $2.88 \pm 0.87$ & $2.90 \pm 0.88$ & 0.0005 \\
\hline FPG (mmol/L) & $6.02 \pm 1.66$ & $5.99 \pm 1.66$ & $5.89 \pm 1.50$ & $<0.0001$ \\
\hline Fasting insulin $(\mu \mathrm{lU} / \mathrm{ml})$ & $6.30(4.40-9.00)$ & $6.80(4.80-9.60)$ & $7.10(5.10-9.90)$ & $<0.0001$ \\
\hline ALT (U/L) & $15.00(11.00-20.00)$ & $16.00(11.00-22.00)$ & $15.00(11.00-22.00)$ & $<0.0001$ \\
\hline AST (U/L) & $21.00(18.00-26.00)$ & $20.00(17.00-25.00)$ & $20.00(16.00-24.00)$ & $<0.0001$ \\
\hline$\gamma-\mathrm{GGT}(\mathrm{U} / \mathrm{L})$ & $20.00(14.00-30.00)$ & $21.00(14.00-32.00)$ & $20.00(14.00-31.90)$ & $<0.0001$ \\
\hline eGFR $\left(\mathrm{mL} / \mathrm{min}\right.$ per $\left.1.73 \mathrm{~m}^{2}\right)$ & $93.65(85.22-99.90)$ & $96.44(87.72-103.23)$ & $96.44(87.19-103.20)$ & $<0.0001$ \\
\hline Ideal physical activity (n (\%)) & $2353(7.84)$ & $4898(13.88)$ & $6684(18.24)$ & $<0.0001$ \\
\hline HOMA-IR & $1.61(1.07-2.44)$ & $1.73(1.18-2.58)$ & $1.80(1.24-2.63)$ & $<0.0001$ \\
\hline Family history of diabetes ( $\mathrm{n}(\%)$ ) & $1722(5.74)$ & $4452(12.61)$ & $6836(18.66)$ & $<0.0001$ \\
\hline
\end{tabular}

Data were means \pm SD or medians (IQRs) for skewed variables or numbers (proportions) for categorical variables.

$P$ for trend was calculated for the linear regression analysis tests across the groups. $P$ values were for analysis of variance or $\chi^{2}$ analyses across the groups.

ALT, alanine aminotransferase ; AST, aspartate aminotransferase ; BMI, body mass index; DBP, diastolic blood pressure; eGFR, estimated glomerular filtration rate; FPG, fasting plasma glucose; HDL-C, high-density lipoprotein cholesterol; HOMA-IR, homeostasis model assessment of insulin resistance; HTN, hypertension; LDL-C, low-density lipoprotein cholesterol; SBP, systolic blood pressure; TC, total cholesterol; TG, triglycerides; WC, waist circumference; $\gamma$-GGT, $\gamma$-glutamyltransferase.

degree or above group were $27.0 \%, 19.4 \%$ and $16.1 \%$, and the incidences of uncontrolled HTN among the education groups were $65.9 \%, 57.8 \%$ and $53.7 \%$, respectively, (table 3 ; all $p$ for trend $<0.0001)$.
The adjusted HRs and 95\% CIs for the risk of newly diagnosed HTN and uncontrolled HTN according to education level are shown in table 3. Compared with the elementary school and below group, the middle school group (unadjusted HR, 0.65;

Table 3 HRs ( $95 \%$ Cls) for incidence of newly diagnosed HTN and uncontrolled HTN according to education level

\begin{tabular}{|c|c|c|c|c|c|}
\hline & & Elementary school and below & Middle school & High school degree or above & $P_{\text {trend }}$ \\
\hline \multirow[t]{6}{*}{ Newly diagnosed HTN } & Incidence (\%) & 27.0 & 19.4 & 16.1 & $<0.0001$ \\
\hline & Model 1 & 1.00 (ref.) & 0.65 (0.63 to 0.68$)$ & $0.51(0.49$ to 0.54$)$ & $<0.0001$ \\
\hline & Model 2 & 1.00 (ref.) & 0.74 (0.70 to 0.77$)$ & 0.59 (0.56 to 0.62$)$ & $<0.0001$ \\
\hline & Model 3 & 1.00 (ref.) & 0.72 (0.68 to 0.75$)$ & $0.58(0.55$ to 0.61$)$ & $<0.0001$ \\
\hline & Model 4 & 1.00 (ref.) & 0.71 (0.67 to 0.74$)$ & $0.58(0.55$ to 0.61$)$ & $<0.0001$ \\
\hline & Model 5 & 1.00 (ref.) & $0.76(0.72$ to 0.80$)$ & $0.67(0.63$ to 0.70$)$ & $<0.0001$ \\
\hline \multirow[t]{6}{*}{ Uncontrolled HTN } & Incidence (\%) & 65.9 & 57.8 & 53.7 & $<0.0001$ \\
\hline & Model 1 & 1.00 (ref.) & 0.83 (0.80 to 0.85$)$ & 0.75 (0.73 to 0.78$)$ & $<0.0001$ \\
\hline & Model 2 & 1.00 (ref.) & $0.82(0.80$ to 0.84$)$ & 0.75 (0.73 to 0.77$)$ & $<0.0001$ \\
\hline & Model 3 & 1.00 (ref.) & 0.81 (0.78 to 0.83$)$ & $0.73(0.71$ to 0.76$)$ & $<0.0001$ \\
\hline & Model 4 & 1.00 (ref.) & 0.84 (0.82 to 0.87$)$ & 0.79 (0.76 to 0.82$)$ & $<0.0001$ \\
\hline & Model 5 & 1.00 (ref.) & 0.90 (0.87 to 0.92$)$ & 0.85 (0.82 to 0.88$)$ & $<0.0001$ \\
\hline
\end{tabular}

Model 1 was unadjusted.

Model 2 was adjusted for age.

Model 3 was adjusted for age, sex and BMI.

Model 4 was adjusted for age, sex, BMI, current smoking, current drinking, family history of diabetes, creatinine, GGT, FPG, insulin, LDL-C and TG.

Model 5 was adjusted for age, sex, BMI, current smoking, current drinking, family history of diabetes, creatinine, GGT, FPG, insulin, LDL-C, TG, baseline SBP and DBP.

BMI, body mass index; DBP, diastolic blood pressure; FPG, fasting plasma glucose; GGT, glutamyltransferase; HTN, hypertension; LDL-C, low-density lipoprotein cholesterol ; SBP, systolic blood pressure; TG, triglycerides. 
$95 \% \mathrm{CI}, 0.63$ to 0.68 ; fully adjusted HR, 0.76 ; $95 \% \mathrm{CI}, 0.72$ to 0.80 ) and high school degree or above group (unadjusted HR, 0.51 ; $95 \% \mathrm{CI}, 0.49$ to 0.54 ; fully adjusted HR, 0.67 ; $95 \% \mathrm{CI}$, 0.63 to 0.70 ) were associated with relatively lower risks of incident HTN. Similarly, compared with the elementary school and below group, the middle school group (unadjusted HR, 0.83; $95 \% \mathrm{CI}, 0.80$ to 0.85 ; fully adjusted HR, $0.90 ; 95 \% \mathrm{CI}, 0.87$ to 0.92 ) or high school degree or above group (unadjusted $\mathrm{HR}$, 0.75 ; $95 \% \mathrm{CI}, 0.73$ to 0.78 ; fully adjusted HR, 0.85 ; 95\% CI, 0.82 to 0.88 ) had lower risks of uncontrolled HTN.

The incidences of newly diagnosed and uncontrolled HTN in the elementary school and below group were higher than those in the middle or above groups. Compared with the elementary school and below group, in the middle or above school groups, the adjusted HR for the risk of newly diagnosed HTN was lower than that of uncontrolled HTN.

\section{Subgroup analyses of education level with newly diagnosed HTN and uncontrolled HTN}

We further explored the influence of educational attainment on blood pressure in subgroup analyses (online supplemental table 1). Consistent results of educational attainment with both newly diagnosed HTN and uncontrolled HTN were observed in various subgroups.

\section{DISCUSSION}

In the present cohort study of 101959 Chinese participants aged 40 years and older, compared with participants at the elementary school education level and below, those with educational attainment at the middle school level or above exhibited a lower risk of newly diagnosed HTN and achieved better blood pressure control in the follow-up phase. The monitoring and management of blood pressure and its control should be strengthened among middle-elderly subjects, especially those with poor educational attainment. The present study, to our best knowledge, is the largest cohort study to investigate associations of educational attainment with the risk of newly diagnosed HTN and uncontrolled HTN.

Elevated blood pressure remains a major public health challenge because it is one of the most crucial risk factors for premature death worldwide. ${ }^{21}$ The prevalence and incidence of HTN has rapidly increased in recent decades in China. ${ }^{22}{ }^{23}$ Overall, in the present study, the prevalent HTN was $44.9 \%$ among adults aged 40 years and older at baseline from 2011 to 2012. In the same age groups, a similar pattern but a much lower rate $(32.2 \%)$ was found among the American population, even though obesity was much more common in the USA from 2011 to $2014 .{ }^{24}$ Disparity of educational attainment could explain the differences in prevalence of HTN among developing and developed countries, even though traditional risk factors for cardiovascular diseases were generally lower in low-income countries. ${ }^{6}$ In a recent nationwide cross-sectional study in China, Zheng et $a l^{11}$ observed a decreased prevalence of HTN and increased HTN control among those with middle and senior education relative to those with only primary education. Accordingly, there is concern about the effects of education levels on the risk of elevated blood pressure in individuals from China, especially among those who have only a below average level of common education. At the same time, information about educational attainment in helping shape the future of blood pressure control remains sparse. We analysed data from the $4 \mathrm{C}$ cohort to conduct nationwide comparisons and found that subjects with higher educational attainment had lower incidence rates of HTN and better blood pressure control in the future.

We showed that education has a marked influence on HTN. On the one hand, this influence is reflected in the increase in newly diagnosed HTN in subjects with elementary school education and below. On the other hand, subjects with elementary school education and below were more likely to have difficulty meeting blood pressure control targets. Subjects with elementary school education and below may be associated with more unhealthy lifestyles, such as an unbalanced diet or a lack of exercise, which are associated with the onset of HTN. According to the German National Health Interview and Examination Survey, Finger et $a l^{25}$ estimated that adults with lower educational attainment consumed calorically dense food more frequently than those with higher educational attainment. Participants with lower education levels are more physically active, which may explain why they consume a high caloric diet. Additionally, patients with HTN with elementary school education and below may show poor compliance with antihypertensive medications. Therefore, our study suggests that the education level of patients with HTN must be considered and the education level of the people as a whole should be increased to avoid HTN and related cerebrovascular diseases.

Our study does have some important limitations. First, the study participants were followed for only a mean of 3.8 years. The relatively short follow-up time could reduce the proportion of new-onset events as well as the statistical power of the study, particularly for evaluating the incidence of HTN. Second, the follow-up rate is important for assessing the validity of the data set for research purposes. However, nearly 12.2\% of study participants were lost to follow-up, which is relatively high and may devastate the reliability of the study results. Third, as the study participants had only one follow-up visit, the blood pressures were evaluated only at the baseline and follow-up visits. This could limit the sensitivity of ascertaining outcomes, especially for the timing of diagnoses for incident HTN. Fourth, the patterns of educational attainment have shown extreme imbalance and differences in regional development in China. Among all of the participants in the study, only 9972 (9.78\%) had a bachelor's degree or higher. Therefore, the findings could not be generalised to all participants worldwide.

In the present Chinese nationwide population-based cohort study, participants with elementary school education levels and below exhibited excess risks of newly diagnosed HTN and worse blood pressure control compared with individuals with educational attainment at the middle school level or above. In our study, the communities included high education and high economy communities, such as Shanghai, Beijing and Guangzhou, while poor economy communities such as Lanzhou were also included. Thus, the study was representative to analyse the effect of educational levels on HTN incidence and control in Chinese populations. Our findings emphasise the value of promoting the overall management of educational attainment in preventing HTN and related cardiovascular events, particularly in countries where education is less accessible.

\section{Author affiliations}

'Department of Endocrinology, Sun Yat-Sen University, Guangzhou, China ${ }^{2}$ Department of Endocrinology, Sun Yat-Sen Memorial Hospital, Guangzhou, China ${ }^{3}$ Shanghai Jiao Tong University Medical School Affiliated Ruijin Hospital, Shanghai, China

${ }^{4}$ Department of Endocrinology, Chinese People's Liberation Army General Hospital, Beijing, China

${ }^{5}$ Department of Endocrinology, Shandong Provincial Hospital, Jinan, Shandong, China 


\section{What is already known on this subject}

- Educational attainment can improve life expectancy and guide healthy behaviours throughout the entire lifetime. A nationwide longitudinal study of the association of the educational status with the risk of hypertension (HTN) and its control in China is lacking.

\section{What this study adds}

- In the present cohort study of 101959 Chinese participants aged 40 years and older, compared with participants at the elementary school education level and below, those with educational attainment at the middle school level or above exhibited a lower risk of newly diagnosed HTN and achieved better blood pressure control in the follow-up phase. Our findings emphasise the importance of promoting the overall management of the educational attainment in the prevention of HTN and related cardiovascular events, particularly in countries where education is less accessible.

${ }^{6}$ Jiangsu Province Hospital on Integration of Chinese and Western Medicine, Nanjing, Jiangsu, China

${ }^{7}$ Union Hospital, Tong-ji Medical College, Hua-zhong University of Science and Technology, Wuhan, China, Wuhan, China

${ }^{8}$ Affiliated Hospital of Guiyang Medical College, Guiyang, China

${ }^{9}$ Second Affiliated Hospital of Harbin Medical University, Harbin, China

${ }^{10}$ Jiangsu Province Hospital and Nanjing Medical University First Affiliated Hospital, Naniing, Jiangsu, China

${ }^{11}$ Affiliated Hospital of Luzhou Medical College, Luzhou, China

${ }^{12}$ Karamay Municipal People's Hospital, Xinjiang, China

${ }^{13}$ Department of Endocrinology, Jilin University First Hospital, Changchun, Jilin, China

${ }^{14}$ Department of Endocrinology, Guangxi Medical University First Affiliated Hospital, Nanning, Guangxi, China

${ }^{15}$ Department of Endocrinology, Lanzhou University First Affiliated Hospital, Lanzhou, Gansu, China

${ }^{16}$ Department of Endocrinology, Fujian Provincial Hospital, Fuzhou, Fujian, China

${ }^{17}$ Department of Endocrinology, Jiangxi Provincial People's Hospital, Nanchang,

Jiangxi, China

${ }^{18}$ Department of Endocrinology, Center Hospital of Dalian, Dalian, China

${ }^{19}$ Department of Endocrinology, Xinhua Hospital Affiliated to Shanghai Jiaotong University School of Medicine, Shanghai, China

${ }^{20}$ Zhejiang Provincial Center for Disease Control and Prevention, Hangzhou, Zhejiang, China

${ }^{21}$ Zhejiang Provincial Center for Disease Control and Prevention, Hangzhou, China

${ }^{22}$ Department of Endocrinology, First Affiliated Hospital of Anhui Medical University, Hefei, China

${ }^{23}$ Department of Endocrinology, Zhengzhou University First Affiliated Hospital, Zhengzhou, Henan, China

${ }^{24}$ Department of Endocrinology, The First Affiliated Hospital of Chongqing Medical University, Chongqing, Sichuan, China

${ }^{25}$ Department of Endocrinology, Huazhong University of Science and Technology، Wuhan, China

${ }^{26}$ Department of Endocrinology, The First Affiliated Hospital of Wenzhou Medical University, Wenzhou, Zhejiang, China

${ }^{27}$ Department of Endocrinology, Shandong University Qilu Hospital, Jinan, Shandong, China

${ }^{28}$ Department of Endocrinology, Shanghai National Research Center for Endocrine and Metabolic Disease, State Key Laboratory of Medical Genomics, Shanghai Institute for Endocrine and Metabolic Disease, Shanghai Jiao Tong University Medical School Affiliated Ruijin Hospital, Shanghai, China

${ }^{29}$ Department of Endocrinology, Sun Yat-Sen Memorial Hospital, Guangzhou, Guangdong, China

Acknowledgements We are indebted to the participants in the present study for their outstanding support and to our colleagues for their valuable assistance.

Contributors $L Y, G N, W Q W$ and KS: Conception of ideas and experimental design. DZL, ML, YMM, JJ Z, CL, YFB, LLC, LXS, QL, TY, QW, SLW, GXW, ZJL, YFQ, XLT, GC,
YNH, ZNG, QS, ZY, RYH, YMW, GJQ, HCD, XFY, FXS and LC: experimentation. KS and ML: data analysis. KS and DZL: Manuscript writing. LY is responsible for the overall content as the guarantor. All authors believe that the manuscript represents valid work and have reviewed and approved the final version.

Funding This work was supported by grants from the following: (1) National Key R\&D Program of China (Nos.2016YFC0901204, 2016YFC1305600, 2016YFC1305202, 2016YFC1304904, 2017YFC1310700, 2018YFC1311705, and 2018YFC1311800); (2) The National Natural Science Foundation of China (81970696); (3) Sun Yat-sen Clinical Research Cultivating Program (SYS-Q-201801); and (4) Sun Yat-sen University Clinical Research 5010 Program (2018021); (5) Science and Technology Planning Project of Guangdong Province, China (2014A020212069); (6) Medical Scientific Research Foundation of Guangdong Province, China (C2019106). These funding associations had no role in the study design, data collection and analysis, decision to publish or preparation of the manuscript.

\section{Competing interests None declared.}

\section{Patient consent for publication Not applicable.}

Ethics approval The protocol of the present study involving human participants was approved by the Institutional Review Board of Ruijin Hospital, Shanghai JiaoTong University School of Medicine and was performed in accordance with the principles of the Helsinki Declaration II.

Provenance and peer review Not commissioned; externally peer reviewed.

Data availability statement Data are available upon reasonable request. Data involved in this study are available upon reasonable request.

Supplemental material This content has been supplied by the author(s). It has not been vetted by BMJ Publishing Group Limited (BMJ) and may not have been peer-reviewed. Any opinions or recommendations discussed are solely those of the author(s) and are not endorsed by BMJ. BMJ disclaims all liability and responsibility arising from any reliance placed on the content. Where the content includes any translated material, BMJ does not warrant the accuracy and reliability of the translations (including but not limited to local regulations, clinical guidelines, terminology, drug names and drug dosages), and is not responsible for any error and/or omissions arising from translation and adaptation or otherwise.

Open access This is an open access article distributed in accordance with the Creative Commons Attribution Non Commercial (CC BY-NC 4.0) license, which permits others to distribute, remix, adapt, build upon this work non-commercially, and license their derivative works on different terms, provided the original work is properly cited, appropriate credit is given, any changes made indicated, and the use is non-commercial. See: http://creativecommons.org/licenses/by-nc/4.0/.

\section{ORCID iDs}

Diaozhu Lin http://orcid.org/0000-0002-8488-5269

Yiming Mu http://orcid.org/0000-0002-3344-3540

Gang Chen http://orcid.org/0000-0002-8105-2384

\section{REFERENCES}

1 Ghaffari S, Roshanravan N. The role of nutraceuticals in prevention and treatment of hypertension: an updated review of the literature. Food Res Int 2020;128:108749.

2 Zlokovic BV, Gottesman RF, Bernstein KE, et al. Vascular contributions to cognitive impairment and dementia (VCID): a report from the 2018 national heart, lung, and blood institute and national institute of neurological disorders and stroke workshop. Alzheimers Dement 2020;16:1714-33.

3 Quiñones J, Hammad Z. Social determinants of health and chronic kidney disease. Cureus 2020;12:e10266.

4 Deere BP, Ferdinand KC. Hypertension and race/ethnicity. Curr Opin Cardiol 2020;35:342-50

5 Doyle SK, Chang AM, Levy P, et al. Achieving health equity in hypertension management through addressing the social determinants of health. Curr Hypertens Rep 2019:21:58.

6 Rosengren A, Smyth A, Rangarajan S, et al. Socioeconomic status and risk of cardiovascular disease in 20 low-income, middle-income, and high-income countries: the prospective urban rural epidemiologic (pure) study. Lancet Glob Health 2019;7:e748-60.

7 Martin A, Johnson AD, Castle S. Reframing high-quality public preschool as a vehicle for narrowing child health disparities based on family income. Acad Pediatr 2021:21:408-13.

8 Nesbitt S, Palomarez RE. Review: increasing awareness and education on health disparities for health care providers. Ethn Dis 2016:26:181-90.

9 Lee H, Nam Y-S, Lee K-M. Development-assistance strategies for stroke in low- and middle-income countries. J Korean Med Sci 2015:30 Suppl 2:S139-42.

10 Wierzejska E, Giernaś B, Lipiak A, et al. A global perspective on the costs of hypertension: a systematic review. Arch Med Sci 2020;16:1078-91.

11 Zheng C, Wang Z, Wang $X$, et al. Social determinants status and hypertension: a nationwide cross-sectional study in China. J Clin Hypertens 2020;22:2128-36. 
12 Yang F, Qian D, Liu X, et al. Socioeconomic disparities in prevalence, awareness, treatment, and control of hypertension over the life course in China. Int J Equity Health 2017;16:100.

13 Li Z, Fu C, Yang F, et al. Prevalence and risk factors of hypertension for the middleaged population in China - results from the China Health and Retirement Longitudinal Study (CHARLS). Clin Exp Hypertens 2019;41:80-6.

14 Wang T, Lu J, Su Q, et al. Ideal cardiovascular health metrics and major cardiovascular events in patients with prediabetes and diabetes. JAMA Cardiol 2019;4:874-83

15 Lu J, He J, Li M, et al. Predictive Value of Fasting Glucose, Postload Glucose, and Hemoglobin $\mathrm{A}_{1 \mathrm{c}}$ on Risk of Diabetes and Complications in Chinese Adults. Diabetes Care 2019;42:1539-48.

16 Tomioka K, Iwamoto J, Saeki K, et al. Reliability and validity of the International physical activity questionnaire (IPAQ) in elderly adults: the Fujiwara-kyo study. J Epidemiol 2011;21:459-65.

17 Levy JC, Matthews DR, Hermans MP. Correct homeostasis model assessment (HOMA) evaluation uses the computer program. Diabetes Care 1998;21:2191-2.

18 Ma Y-C, Zuo L, Chen J-H, et al. Modified glomerular filtration rate estimating equation for Chinese patients with chronic kidney disease. J Am Soc Nephrol 2006;17:2937-44.
19 Chobanian AV, Bakris GL, Black HR, et al. Seventh report of the joint National Committee on prevention, detection, evaluation, and treatment of high blood pressure. Hypertension 2003;42:1206-52.

20 Crim MT, Yoon SSS, Ortiz E, et al. National surveillance definitions for hypertension prevalence and control among adults. Circ Cardiovasc Qual Outcomes 2012;5:343-51.

21 GBD 2017 Risk Factor Collaborators. Global, regional, and national comparative risk assessment of 84 behavioural, environmental and occupational, and metabolic risks or clusters of risks for 195 countries and territories, 1990-2017: a systematic analysis for the global burden of disease study 2017. Lancet 2018;392:1923-94.

22 Liu L, Chen C-L, Lo K, et al. Trends of status of hypertension in southern China, 20122019. Int J Gen Med 2020;13:599-608.

23 Liu W, Leong DP, Hu B, et al. The association of grip strength with cardiovascular diseases and all-cause mortality in people with hypertension: findings from the prospective urban rural epidemiology China study. J Sport Health Sci 2020. doi:10.1016/j.jshs.2020.10.005. [Epub ahead of print: 19 Oct 2020].

24 Yoon SSS, Carroll MD, Fryar CD. Hypertension prevalence and control among adults: United States, 2011-2014. NCHS Data Brief 2015:1-8.

25 Finger JD, Tylleskär T, Lampert T, et al. Dietary behaviour and socioeconomic position: the role of physical activity patterns. PLoS One 2013;8:e78390. 